\title{
Tractable stability analysis for systems containing repeated scalar slope-restricted nonlinearities
}

\author{
Matthew C. Turner ${ }^{1, *, \dagger}$, Murray L. Kerr ${ }^{2}$ and Jorge Sofrony ${ }^{3}$ \\ ${ }^{1}$ Control and Instrumentation Research Group, Department of Engineering, University of Leicester, Leicester, \\ LE1 $7 R H, U K$ \\ ${ }^{2}$ Deimos Space SL, Madrid, 28760, Spain \\ ${ }^{3}$ Department of Mechatronics, National University of Colombia, Bogota, Colombia
}

\begin{abstract}
SUMMARY
This paper proposes an LMI-based approach for studying the stability of feedback interconnections of a finite dimensional LTI system and a nonlinear element that consists of several identical scalar nonlinearities that have restrictions on their sector and slope. The results are based on the integral quadratic constraint stability analysis framework and other recent results that give a sharp characterisation of stability multipliers for monotone, repeated scalar nonlinearities. Several examples show the effectiveness of the proposed approach; the lack of conservatism in the results is noteworthy. Copyright @ 2013 John Wiley \& Sons, Ltd.
\end{abstract}

Received 24 June 2013; Revised 25 October 2013; Accepted 30 October 2013

KEY WORDS: absolute stability; slope-restricted nonlinearity; sector bounds; integral quadratic constraints; anti-windup

\section{INTRODUCTION}

This paper re-visits the much-studied stability problem depicted in Figure 1 in which $P(s)$ is a finite dimensional LTI system and $\Phi():. \mathcal{L}_{2 e}^{m} \mapsto \mathcal{L}_{2 e}^{\mathrm{m}}$ is a static time-invariant nonlinear element. Depending on the information available about $\Phi($.$) , different criteria may be used to study the sta-$ bility of the interconnection. Commonly, the Circle and Popov criteria are used to assess the stability of such systems, but when further information about the nonlinearity is known, and depending on the computational resources available, various other criteria may be used instead. In order to keep the paper reasonably succinct, we do not review all available approaches, but the interested reader is encouraged to consult [1-6] and references therein.

Of all the various stability criteria available for the study of the system in Figure 1, one of the most promising was proposed in the late 1960s by Zames and Falb [7]. Zames and Falb proved that

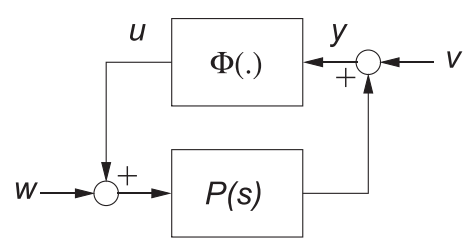

Figure 1. System under consideration.

\footnotetext{
*Correspondence to: Matthew C. Turner, Control and Instrumentation Research Group, Department of Engineering, University of Leicester, Leicester, LE1 7RH, UK.

†E-mail: mct6@le.ac.uk 
if $\Phi($.$) was a scalar valued nonlinearity, which was either (i) monotone non-decreasing and within$ some finite sector or (ii) its slope was restricted to lie in a given interval, then stability could be examined using a particular type of multiplier. In essence, reference [7] showed that a certain class of multipliers - the so-called Zames-Falb multipliers $M(s) \in \mathcal{M}_{Z F}$ - were positivity preserving: that is, the inner product $\langle M, \Phi\rangle \geqslant 0$ when $M(s) \in \mathcal{M}_{Z F}$. In the causal case, this meant that if one could establish passivity of $M(s) P(s)$, then the system in Figure 1 would be stable. This result is an obvious improvement over standard passivity results, which do not incorporate multipliers, and allows one to prove stability of a much larger class of systems (see [6] for more information on the use of multipliers in stability analysis). In particular, stability analysis carried out using Zames-Falb multipliers, when appropriate, is often significantly less conservative than that carried out using the Popov or Circle criteria, making the Zames-Falb multipliers an appealing analysis tool.

The main drawback of the work of reference [7] was that to obtain the least conservative results, in the sense that stability held for the largest class of $\Phi($.$) , one had to search over the whole class$ of $\mathcal{M}_{Z F}$, which constitutes an infinite-dimensional optimisation problem. A further drawback was that the original results in [7] were only derived for scalar nonlinearities, $\Phi():. \mathcal{L}_{2 e} \mapsto \mathcal{L}_{2 e}$ and although multivariable extensions were envisaged, it was not until Safonov's more recent work [8] that these were correctly proved.

For the aforementioned reasons, although the Zames-Falb multipliers were a breakthrough in stability analysis, their use in mainstream control engineering has been fairly limited since their introduction. Recently, however, researchers have found renewed interest in stability analysis using Zames-Falb multipliers. Research pioneered by Safonov and co-authors has considerably improved the understanding of such multipliers in the multivariable case, extending the results of [7] to the general MIMO case [8], then to repeated scalar nonlinearities [9] and finally to repeated MIMO nonlinearities [10]. In each case, Safonov and colleagues derived the largest class of Zames-Falb-type multipliers that preserved positivity of $\langle M, \Phi\rangle$. Recently, the work of $[8,9]$ has also been extended to allow the nonlinearity to be static and time periodic [11].

In tandem with these theoretical results, the late 1990s saw the introduction of integral quadratic constraint (IQC)-based analysis [12,13], which gave a common framework for stability analysis problems involving different nonlinearities and in which the Zames-Falb multipliers played a significant role. Moreover, the work presented in [13] was able to provide an IQC framework for Zames-Falb multipliers for repeated scalar nonlinearities, and although, as proved in [9], such a class was actually more limited than necessary, this represented a major step forward in the computation of such multipliers. However, even in [13] (and [12]), the Zames-Falb multipliers were still chosen in a relatively ad hoc manner: in essence, a simple multiplier structure satisfying the appropriate Zames-Falb conditions $M(s) \in \mathcal{M}_{Z F}$ was selected, parameters were then chosen in an ad hoc manner, and finally, stability was checked via IQC tools. Although such an approach can be effective for simple systems and offers improvement over many other techniques, such ad hoc choices of multiplier parameters is not satisfactory in general.

This lack of guidance in multiplier construction was addressed to some extent in [14] where it was shown, for SISO systems, that when the Zames-Falb multipliers were restricted to have a special structure - that is, to be causal and to have order equal to the LTI part of the interconnection - the stability of such systems could be cast as an LMI feasibility problem together with a line search. The rationale behind this work was to obtain tractability by restricting the class of multipliers over which one searched. Thus, while in principle the price paid for tractability was conservatism, it was shown in [14-16] that, despite this restriction in the class of multipliers, the results were encouraging and, for slope-restricted nonlinearities, were able, in some cases, to outperform the Lyapunov-based methods of [4]. Related results [17] in which the multiplier was restricted to be anti-causal were shown, in some cases, to lessen conservatism further. Further results, using a different approach, have recently been contributed in [18].

The obvious weakness of the results in [14] is their limited applicability, because only SISO systems were considered. The goal of this paper is to extend the results of [14] to a class of MIMO systems, namely systems in which $\Phi($.$) takes the form of a repeated scalar nonlinearity. As argued$ in [13] and [9], although such a condition on $\Phi($.$) seems restrictive, simple 'loop shifting' (see [19])$ can often be employed to manipulate the system nonlinearities into such a form. In this paper, it 
will be assumed that the repeated nonlinearities are both slope restricted and sector bounded, in a similar way to [16]. While it is easy to see that slope restriction implies a sector bound of the same size, it may be the case that the nonlinearity in question satisfies a 'tighter' sector bound and thus information about this sector bound can be used to reduce conservatism in the results. While the development of the MIMO results takes inspiration from the scalar case, the precise details change in order to cope with the multivariable nature of the problem, and in particular, the results appeal to the recent work of [9] in order to obtain appropriate conditions on the Zames-Falb multiplier. The paper also uses the IQC machinery to develop the results and, from one perspective, may be viewed as an improvement on the work of [13] in which the multiplier is chosen more efficiently and from the larger set proposed by [9]. A preliminary version of this paper appeared in [20].

Notation: Notation is standard throughout. $H_{i j}$ denotes the $i j$ 'th element of the matrix $H ; H_{i}$ denotes the $i$ 'th row/column of the matrix $H$ (meaning discernible from context). The $\mathcal{L}_{2}$ norm of a vector-valued function $x(t)$ is defined as $\|x\|_{2}:=\sqrt{\int_{0}^{\infty}\|x(t)\|^{2} d t}$ where $\|x\|$ denotes a vector's Euclidean norm. The space of $m$-dimensional vector-valued functions with finite $\mathcal{L}_{2}$ norm is denoted by $\mathcal{L}_{2}^{m}$ and the extended space $\mathcal{L}_{2 e}^{m}$. In instances when the dimension of the space is not important, we simply write $x \in \mathcal{L}_{2}$ and $x \in \mathcal{L}_{2 e}$, respectively. Similarly, the $\mathcal{L}_{1}$ norm of a scalarvalued function $\delta(t)$ is defined as $\|\delta\|_{1}:=\int_{0}^{\infty}\|\delta(t)\| d t$, and the space where this norm is finite is denoted by $\mathcal{L}_{1}$. With some abuse of notation, we say that a transfer function matrix $H(s) \in \mathcal{L}_{1}$ if the impulse responses of its elements $H_{i j}(t)$ are all in $\mathcal{L}_{1}$. The space of real rational $m \times n$ transfer function matrices, bounded on the imaginary axis, is denoted by $\mathcal{R} \mathcal{L}_{\infty}^{m \times n}$; the subspace of $\mathcal{R} \mathcal{L}_{\infty}^{m \times n}$, which is analytically continuous in the right half complex plane, is denoted by $\mathcal{R} \mathcal{H}_{\infty}^{m \times n}$. An operator $H$ is said to be bounded if $\|H(u)\| \leqslant \gamma\|u\|$ for all $u \in \mathcal{L}_{2 \mathrm{e}}$ and some $\gamma>0$.

\section{NONLINEARITIES AND INTEGRAL QUADRATIC CONSTRAINTS}

Consider Figure 1 in which $P(s)$ is the finite dimensional LTI part of the system with state-space realisation

$$
P(s) \sim\left[\begin{array}{c|c}
A_{p} & B_{p} \\
\hline C_{p} & D_{p}
\end{array}\right]
$$

where $A_{p} \in \mathbb{R}^{n \times n}, B_{p} \in \mathbb{R}^{n \times m}, C_{p} \in \mathbb{R}^{m \times n}$ and $D_{p} \in \mathbb{R}^{m \times m}$. In this paper, we assume that $\Phi($.) belongs to the class of so-called repeated static scalar nonlinearities, $\mathcal{N}^{R S}$, defined below.

Definition 1 (Repeated scalar nonlinearity)

$\Phi():. \mathcal{L}_{2 e}^{m} \mapsto \mathcal{L}_{2 e}^{m} \in \mathcal{N}^{R S}$ if

(i) It is memoryless and has the form

$$
\Phi(.)=\left[\begin{array}{c}
\phi(.) \\
\phi(.) \\
\vdots \\
\phi(.)
\end{array}\right] \quad \phi(.): \mathbb{R} \mapsto \mathbb{R}
$$

(ii) $\|\phi(u)\|<\gamma\|u\|$ for all $u \in \mathcal{L}_{2 e}$

(iii) $\phi(0)=0$

(iv) $\phi(u)=-\phi(-u)$

The oddness assumption (meaning the nonlinearity is symmetric) in item (iv) is actually not necessary but will be assumed here for brevity of presentation. This paper will consider several nonlinearities belonging to this class. Of particular interest are the so-called sector bounded nonlinearities, where a static nonlinearity $\phi($.$) is said to belong to the sector [0, \beta](\phi(.) \in \operatorname{Sector}[0, \beta])$ if, for some $\beta>0$, the following inequality holds.

$$
0 \leqslant \phi(x) x \leqslant \beta x^{2} \quad \forall x \in \mathbb{R}, \quad \beta>0
$$


Similarly, $\phi($.$) is said to have slope restriction [0, \alpha](\phi(.) \in \partial[0, \alpha])$ if, for some $\alpha>0$, the following inequality holds.

$$
0 \leqslant \frac{\phi(x)-\phi(y)}{x-y} \leqslant \alpha \quad \forall x, y \in \mathbb{R}, \quad \alpha>0
$$

It is straightforward to see that $\phi(.) \in \partial[0, \alpha] \Rightarrow \phi(.) \in \operatorname{Sector}[0, \alpha]$, but the converse is not true. It may be that a nonlinearity satisfying a certain slope restriction, $\partial[0, \alpha]$, may satisfy tighter sector bounds, that is, $\phi(.) \in \operatorname{Sector}[0, \beta]$, with $\beta<\alpha$. It is emphasised that many nonlinearities that are encountered in practice are both sector and slope bounded; the saturation and deadzone nonlinearities are two common examples. For such nonlinearities, the sector and slope bounds are the same, but this is not always the case.

Finally, a nonlinearity $\phi($.$) is said to be monotonically non-decreasing if the following$ inequality holds:

$$
(\phi(x)-\phi(y))(x-y) \geqslant 0 \quad \forall x, y \in \mathbb{R}
$$

The classes of nonlinearity considered in the remainder of the paper are now formally introduced.

\section{Definition 2 (Sector-bounded/slope-restricted nonlinearities)}

1. Sector-bounded nonlinearity: $\Phi(.) \in \mathcal{N}_{* / \beta}^{R S}$ if $\Phi(.) \in \mathcal{N}^{R S}$, and $\phi(.) \in \operatorname{Sector}[0, \beta], \beta>0$.

2. Slope-restricted nonlinearity: $\Phi(.) \in \mathcal{N}_{\alpha / *}^{R S}$ if $\Phi(.) \in \mathcal{N}^{R S}$, and $\phi(.) \in \partial[0, \alpha], \alpha>0$.

3. Sector-bounded/slope-restricted: $\Phi(.) \in \mathcal{N}_{\alpha / \beta}^{R S}$ if $\Phi(.) \in \mathcal{N}_{\alpha / *}^{R S} \cap \mathcal{N}_{* / \beta}^{R S}$.

4. Monotone non-decreasing nonlinearity: $\Phi(.) \in \mathcal{N}_{M}^{R S}$ if $\Phi(.) \in \mathcal{N}^{R S}, \Phi(.) \in \mathcal{N}_{* / \beta}^{R S}$ for some $\beta \in(0, \infty)$ and $\phi($.$) is monotonically non-decreasing.$

Remark 1

$\mathcal{N}_{* / \beta}^{R S}$ is simply the class of repeated scalar nonlinearities in which each scalar nonlinearity is sector bounded with the same bound. It is easy to see that typical nonlinearities such as multivariable saturations/deadzones can be 'loop shifted' into this form. Likewise, $\mathcal{N}_{\alpha / *}^{R S}$ is the set of repeated scalar nonlinearities in which each entry is slope restricted by the same bound. Again, many common nonlinearities can be 'loop shifted' to be in this form. A sub-class of $\mathcal{N}_{\alpha / *}^{R S}$ was studied in [21]. $\mathcal{N}_{\alpha / \beta}^{R S}$ is simply the intersection of the two previous classes of nonlinearities. Note that, trivially, $\mathcal{N}_{\alpha / *}^{R S}=\mathcal{N}_{\alpha / \alpha}^{R S}$; that is, the slope restriction implies the same sector bound. However, it is possible for the sector bound to be smaller than the slope restriction; that is, $\mathcal{N}_{\alpha / \beta}^{R S} \subset \mathcal{N}_{\alpha / *}^{R S}=\mathcal{N}_{\alpha / \alpha}^{R S}$ in general; that is, using $\beta$ may reduce conservatism.

\section{Remark 2}

$\mathcal{N}_{M}^{R S}$ is basically the class of monotone non-decreasing nonlinearities considered in [7] but generalised to the repeated scalar case ([9]). The significance of this class of nonlinearities is that it can be proved, under mild conditions, that if a nonlinearity $\Phi(.) \in \mathcal{N}_{\alpha / *}^{R S}$, then loop shifting can be used to transform it into an equivalent nonlinearity of the form $\tilde{\Phi}(.) \in \mathcal{N}_{M}^{R S}$, effectively meaning that stability results available for the class $\mathcal{N}_{M}^{R S}$ can be used for the class $\mathcal{N}_{\alpha / *}^{R S}$. In our work, this means that the new Zames-Falb multipliers derived by [9] can be applied to the class of nonlinearities considered in this paper. This is important because it allows us to obtain results of reduced conservatism compared with [13] and in fact is easier to use in obtaining convex conditions. See [6] for a more comprehensive discussion of loop shifting.

The main objective of this paper is to obtain tractable results that can be used to assess the stability of the feedback interconnection described by Figure 1. In order to do this, extensive use is made of 
the IQC analysis framework introduced in $[5,12]$ and extended in $[13,22]$. In general, it is said that a nonlinearity $\Phi($.$) satisfies the IQC defined by \Pi(j \omega)$ if $\Phi$ is such that

$$
\int_{-\infty}^{\infty}\left[\begin{array}{l}
\hat{y}(j \omega) \\
\hat{u}(j \omega)
\end{array}\right]^{*} \Pi(j \omega)\left[\begin{array}{l}
\hat{y}(j \omega) \\
\hat{u}(j \omega)
\end{array}\right] d \omega \geqslant 0
$$

where $\hat{y}(j \omega)$ and $\hat{u}(j \omega)$ are the Fourier Transforms of $y(t)$ and $u(t)=\Phi(y(t))$, respectively. The structure of $\Pi(j \omega)$ depends on the properties of the nonlinearity $\Phi($.$) . There are several different$ IQCs that will be used in this paper.

1. The Zames-Falb IQC. The 'Zames-Falb' IQC used here is a combination of the one presented in [13] with the relaxation suggested by [9]. More precisely, if $\Phi(.) \in \mathcal{N}_{\alpha / *}^{R S}$, it satisfies the IQC defined by $\Pi=\Pi_{\alpha}^{Z F}(j \omega)$ where

$$
\Pi_{\alpha}^{Z F}(j \omega)=\left[\begin{array}{cc}
0 & \alpha M^{*}(j \omega) \\
\alpha M(j \omega) & -M^{*}(j \omega)-M(j \omega)
\end{array}\right]
$$

and $M(s)$ is a multiplier such that

$$
M(s)=H_{0}-H(s) \in \mathcal{R} \mathcal{L}_{\infty}^{m \times m}
$$

where

$$
\begin{aligned}
& H_{0, i i} \geqslant \sum_{j=1, j \neq i}^{m}\left|H_{0, i j}\right|+\left\|H_{i j}\right\|_{1} \quad \forall i \in\{1, \ldots, m\} \\
& H_{0, i i} \geqslant \sum_{j=1, j \neq i}^{m}\left|H_{0, j i}\right|+\left\|H_{j i}\right\|_{1} \quad \forall i \in\{1, \ldots, m\}
\end{aligned}
$$

This class of multiplier, $M(s)$, is somewhat broader than that in [13] because the improvement suggested by [9] does not require the multiplier $M(s)$ to be symmetric: only the row and column dominance conditions in (5) and (6) are stipulated. This improvement is both important in lessening conservatism of the results and also in allowing our new results to be cast as a quasi-convex optimisation problem.

2. The Circle IQC. It is well known (see, e.g. [5]) that if $\Phi(.) \in \mathcal{N}_{* / \beta}^{R S}$, it satisfies the IQC given by $\Pi=\Pi_{\beta}^{C}$, where

$$
\Pi_{\beta}^{C}(j \omega)=\left[\begin{array}{cc}
0 & \beta V \\
\beta V & -2 V
\end{array}\right]
$$

and $V$ is any positive definite diagonal matrix. Note that because $\Phi \in \mathcal{N}_{\alpha / *}^{R S} \Longrightarrow \Phi \in \mathcal{N}_{* / \alpha}^{R S}$ (actually $\Phi \in \mathcal{N}_{\alpha / \alpha}^{R S}$ ), this multiplier can be used with any slope restricted nonlinearity.

3. The Popov IQC. Following [22], if $\Phi(.) \in \mathcal{N}_{* / \beta}^{R S}$, it satisfies the IQC given by $\Pi=\Pi^{P}$ where

$$
\Pi^{P}(j \omega)=\left[\begin{array}{cc}
0 & (j \omega \Lambda)^{*} \\
j \omega \Lambda & 0
\end{array}\right]
$$

where $\Lambda$ is an (indefinite) diagonal matrix. As with the Circle IQC, the Popov IQC can also be used in the analysis of slope-restricted nonlinearities.

One of the attractive features of IQC analysis $[5,12,13]$ is that if a nonlinearity satisfies more than one IQC, these IQCs can be combined. Hence, when $\Phi \in \mathcal{N}_{\alpha / \beta}^{R S}$, it satisfies the IQC defined by $\Pi_{\alpha / \beta}^{R S}:=\Pi_{\alpha}^{Z F}+\Pi^{P}+\Pi_{\beta}^{C}$, which is given later.

$$
\Pi_{\alpha / \beta}^{R S}(j \omega):=\left[\begin{array}{cc}
0 & (\beta V+j \omega \Lambda+\alpha M(j \omega))^{*} \\
\beta V+j \omega \Lambda+\alpha M(j \omega) & -2 V-M^{*}(j \omega)-M(j \omega)
\end{array}\right]
$$


where $M(s)=H_{0}-H(s)$ such that inequalities (5) and (6) are satisfied, $\Lambda$ is a diagonal matrix and $V$ is a positive definite diagonal matrix. Theorem 1 of [22] then allows the following theorem to be stated.

\section{Theorem 1}

Consider the interconnection in Figure 1 where $P(s) \in \mathcal{R} \mathcal{H}_{\infty}$ and $\Phi():. \mathcal{L}_{2 e}^{m} \mapsto \mathcal{L}_{2 e}^{m} \in \mathcal{N}_{\alpha / \beta}^{R S}$ for some $\alpha>0$ and $\beta>0$. Assume that the closed-loop system is well posed. Then, the system is stable if the following inequality is satisfied.

$$
\left[\begin{array}{c}
P(j \omega) \\
I
\end{array}\right]^{*} \Pi_{\alpha / \beta}^{R S}(j \omega)\left[\begin{array}{c}
P(j \omega) \\
I
\end{array}\right]<0 \quad \forall \omega \in \mathbb{R}
$$

\section{Remark 3}

Strictly speaking, 'stable' here means that $\mathcal{L}_{2}$ boundedness of the exogenous signals, $w$ and $v$, implies $\mathcal{L}_{2}$ boundedness of all loop signals; that is, 'stability' of Figure 1 means ' $\mathcal{L}_{2}$ stability' of Figure 1. However, as $P(s) \in \mathcal{R} \mathcal{H}_{\infty}^{m \times m}$ and $\Phi($.$) is static, \mathcal{L}_{2}$ stability of Figure 1 actually implies global asymptotic stability of the origin of the unforced system.

\section{Remark 4}

In this form, Theorem 1 is quite difficult to verify efficiently; one needs to ensure inequality (10) holds for $M(s)$ of a very general structure. In $[12,13,23]$, the approach advocated has been to fix a structure of $M(s)$ such that inequalities (5) and (6) hold and then to check inequality (10); no real guidance on how to choose $M(s)$ was given, and the results in [13] basically reduce to the checking of ad hoc structures for $M(s)$, which, although possible and useful for some systems, is rather time consuming in general. In $[14,16]$, it was shown how, by restricting the structure of $M(s)$ to be causal and of order equal to that of $P(s)$, it was possible to choose $M(s)$ much more systematically using LMIs and a line search. Those results were confined to the case of SISO systems; here, we generalise those results to MIMO systems with repeated scalar nonlinearities.

\section{MAIN RESULTS}

The aim of this section is to provide a tractable way of determining whether the system in Figure 1 is stable when $\Phi \in \mathcal{N}_{\alpha / \beta}^{R S}$. If the sector information is discarded, that is, if $\Phi \in \mathcal{N}_{\alpha / *}^{R S}$ and the Popov and Circle IQC's are ignored, the results will reduce to a multivariable generalisation of those in [14]. Related results will be discussed after the main result has been presented

\subsection{Preliminary results}

There are a number of preliminary results required for proving the main results.

Fact 1

$\Pi_{\alpha / \beta}(j \omega)$ can be factorised as $\Pi_{\alpha / \beta}(j \omega)=U^{*}(j \omega) W U(j \omega)$ where

$$
\begin{aligned}
U(j \omega) & :=\left[\begin{array}{cc}
-\alpha I & I \\
0 & I \\
-\alpha H(j \omega)+\beta V+j \omega \Lambda & H(j \omega)-V \\
0 & -I
\end{array}\right] \\
W & :=\left[\begin{array}{cccc}
0 & -H_{0}^{\prime} & 0 & 0 \\
-H_{0} & 0 & 0 & 0 \\
0 & 0 & 0 & -I \\
0 & 0 & -I & 0
\end{array}\right]
\end{aligned}
$$


Proof

Follows by direct calculation.

\section{Lemma 1}

Consider a transfer function matrix $H(s) \in \mathcal{R} \mathcal{H}_{\infty}^{m \times m}$. Then, $\left\|H_{i j}\right\|_{1} \leqslant \xi_{i j}$ for some $i, j$ if there exist positive definite matrices $Y_{j}>0$ and scalars $\mu_{j}>0$ and $\lambda_{j}>0$ such that the following matrix inequalities hold for all $i, j \in\{1, \ldots, m\}$ :

$$
\begin{gathered}
{\left[\begin{array}{cc}
A_{H}^{\prime} Y_{j}+Y_{j} A_{H}+\lambda_{j} Y_{j} & Y_{j} B_{H, j} \\
\star & -\mu_{j}
\end{array}\right]<0} \\
{\left[\begin{array}{ccc}
\lambda_{j} Y_{j} & 0 & C_{H, i}^{\prime} \\
\star & \left(\xi_{i j}-\mu_{j}\right) & D_{H, i j}^{\prime} \\
\star & \star & \xi_{i j}
\end{array}\right] \geqslant 0}
\end{gathered}
$$

\section{Proof}

This result is a slight generalisation of that proved in [24] - see appendix for proof.

\section{Lemma 2}

Consider the real matrix $H_{0} \in \mathbb{R}^{m \times m}$ and positive scalars $\gamma_{i}>0 \quad \forall i \in\{1, \ldots, m\}$. The inequalities

$$
H_{0, i i} \geqslant \sum_{j=1, j \neq i}^{m}\left|H_{0, i j}\right|+\gamma_{i} \quad \forall i \in\{1, \ldots, m\}
$$

hold if and only if there exists a real matrix $R \in \mathbb{R}^{m \times m}$ such that

1. $R_{i j} \geqslant 0 \quad \forall i \neq j$

2. $H_{0, i j}+R_{i j} \geqslant 0 \quad \forall i \neq j$

3. $H_{0, i i} \geqslant \sum_{j=1, j \neq i}^{m} H_{0, i j}+2 R_{i j}+\gamma_{i} \quad \forall i \in\{1, \ldots, m\}$

\section{Proof}

This lemma is a non-symmetric generalisation of a result of [21]. Symmetry is not required, and the proof follows easily from that in [21]. Note that this result allows an inequality involving a nonlinear function of a matrix variable $H_{0}$ to be verified by a set of linear conditions given in items $1-3$.

\subsection{Main result}

The following is the main result of the paper.

\section{Theorem 2}

Assume $P(s) \in \mathcal{R} \mathcal{H}_{\infty}^{m \times m}$ with $D_{p} \equiv 0$ and that $\Phi(.) \in \mathcal{N}_{\alpha / \beta}^{\mathrm{RS}}$. Then, the system in Figure 1 is stable if there exist positive definite matrices $\mathbf{S}_{\mathbf{1 1}}, \mathbf{P}_{\mathbf{1 1}}$, a positive definite diagonal matrix $\mathbf{V}$, a diagonal matrix $\boldsymbol{\Lambda}$, positive scalars $\lambda_{j}, \mu_{j}, \xi_{i j}$, and unstructured matrices $\mathbf{A}_{\mathbf{H}}, \mathbf{B}_{\mathbf{H}}, \mathbf{C}_{\mathbf{H}}$ and $\mathbf{D}_{\mathbf{H}}, \mathbf{H}_{0}$ and $\mathbf{R}$ such that the following inequalities hold:

$$
\begin{aligned}
& {\left[\begin{array}{ccc}
\mathbf{S}_{11} A_{p}+A_{p}^{\prime} \mathbf{S}_{11} & \mathbf{S}_{11} A_{p}+A_{p}^{\prime} \mathbf{P}_{11}-\alpha C_{p}^{\prime} \mathbf{B}_{\mathbf{H}}^{\prime}+\mathbf{A}_{\mathbf{H}}^{\prime} & \mathbf{S}_{11} B_{p}+\alpha C_{p}^{\prime}\left(\mathbf{H}_{0}-\mathbf{D}_{\mathbf{H}}\right)^{\prime}+\mathbf{C}_{\mathbf{H}}^{\prime}+\beta C_{p}^{\prime} \mathbf{V}+A_{p}^{\prime} C_{p}^{\prime} \mathbf{\Lambda} \\
\star & A_{p}^{\prime} \mathbf{P}_{11}+\mathbf{P}_{11} A_{p}-\mathbf{B}_{\mathbf{H}} \alpha C_{p}-\alpha C_{p}^{\prime} \mathbf{B}_{\mathbf{H}}^{\prime} & \mathbf{P}_{11} B_{p}+\mathbf{B}_{\mathbf{H}}+\alpha C_{p}^{\prime}\left(\mathbf{H}_{0}-\mathbf{D}_{\mathbf{H}}\right)^{\prime}+\beta C_{p}^{\prime} \mathbf{V}+A_{p}^{\prime} C_{p}^{\prime} \mathbf{\Lambda} \\
\star & \star & \left(\mathbf{D}_{\mathbf{H}}-\mathbf{H}_{0}\right)^{\prime}+\left(\mathbf{D}_{\mathbf{H}}-\mathbf{H}_{0}\right)-2 \mathbf{V}+\mathbf{\Lambda} C_{p} B_{p}+B_{p}^{\prime} C_{p}^{\prime} \mathbf{\Lambda}
\end{array}\right]<0} \\
& {\left[\begin{array}{cc}
-\mathbf{A}_{\mathbf{H}}-\mathbf{A}_{\mathbf{H}}^{\prime}+\lambda_{j}\left(\mathbf{P}_{\mathbf{1 1}}-\mathbf{S}_{\mathbf{1 1}}\right) & \mathbf{B}_{\mathbf{H}, \mathbf{j}} \\
\star & -\mu_{j}
\end{array}\right]<0 \forall j \in 1, \ldots, m}
\end{aligned}
$$




$$
\begin{gathered}
{\left[\begin{array}{ccc}
-\lambda_{j}\left(\mathbf{S}_{\mathbf{1 1}}-\mathbf{P}_{\mathbf{1 1}}\right) & 0 & \mathbf{C}_{\mathbf{H , i}}{ }^{\prime} \\
\star & \xi_{i j}-\mu_{j} & \mathbf{D}_{\mathbf{H}, \mathbf{j}} \\
\star & \xi_{i j}
\end{array}\right]>0 \forall i, j \in 1, \ldots, m} \\
\mathbf{H}_{0, i i} \geqslant \sum_{j=1, j \neq i}^{m} \mathbf{H}_{0, i j}+2 \mathbf{R}_{i j}+\sum_{j=1}^{m} \xi_{i j} \forall i \in 1, \ldots, m \\
\mathbf{H}_{0, i i} \geqslant \sum_{j=1, j \neq i}^{m} \mathbf{H}_{0, j i}+2 \mathbf{R}_{j i}+\sum_{j=1}^{m} \xi_{j i} \forall i \in 1, \ldots, m \\
\mathbf{R}_{i j} \geqslant 0 \quad \forall i, j \in 1, \ldots, m \\
\mathbf{H}_{0, i j}+\mathbf{R}_{i j} \geqslant 0 \quad \forall i, j \in 1, \ldots, m
\end{gathered}
$$

\section{Remark 5}

For fixed $\alpha>0, \beta>0$ and $\lambda_{j}>0$ for all $j \in\{1, \ldots, m\}$, conditions (16)-(22) form a system of linear matrix inequalities, which can be solved efficiently using modern software. Thus, if the sector/slope bounds are known a priori, it is relatively easy to verify stability. If, instead, it is necessary to compute the maximum sector/slope size for which the system remains stable, this can be performed by combining the LMIs in (16)-(22) with a bisection over $\alpha$ (fixing $\beta=\kappa \alpha$ for some constant $\kappa \in[0,1])$ and a further search over $\lambda_{j}$. The key point is that Theorem 2 gives a relatively efficient, if potentially conservative, way of determining the stability of Figure 1.

\subsection{Proof of Theorem 2}

The proof of Theorem 2 basically requires us to translate the frequency domain inequality in Theorem 1 and the $\mathcal{L}_{1}$ constraints in inequalities (5) and (6) into matrix inequalities. There are three distinct parts of the proof, which, as will be shown, are coupled through some matrix variables.

Part 1: Frequency domain condition

It is necessary to convert the frequency domain inequality in Theorem 1 into a set of matrix inequalities. This part of the proof is shared with that reported in $[14,15]$, and so it will only be sketched. First note that inequality (10) can be written

$$
\left[\begin{array}{c}
P(j \omega) \\
I
\end{array}\right]^{*} U(j \omega)^{*} W U(j \omega)\left[\begin{array}{c}
P(j \omega) \\
I
\end{array}\right]<0 \quad \forall \omega \in \mathbb{R}
$$

where $U(j \omega)$ and $W$ are given in Fact 1. Assuming that $H(s)$ is causal, of order equal to $P(s)$ and has state-space realisation $H(s) \sim\left(A_{H}, B_{H}, C_{H}, D_{H}\right)$, it follows that a state-space realisation of $U(s)\left[P(s)^{\prime} \quad I\right]^{\prime} \sim(\bar{A}, \bar{B}, \bar{C}, \bar{D})$ is given by

$$
\left[\begin{array}{c|c}
\bar{A} & \bar{B} \\
\hline \bar{C} & \bar{D}
\end{array}\right]:=\left[\begin{array}{cc|c}
A_{p} & 0 & B_{p} \\
-\alpha B_{H} C_{p} & A_{H} & B_{H} \\
\hline-\alpha C_{p} & 0 & I \\
0 & 0 & I \\
-\alpha D_{H} C_{p}+\beta V C_{p}+\Lambda C_{p} A_{p} & C_{H} & D_{H}+\Lambda C_{p} B_{p}-V \\
0 & 0 & -I
\end{array}\right]
$$

Using this state-space realisation and the KYP Lemma [25], it then follows that inequality (23) is satisfied (and hence, inequality (10) is satisfied) if there exists a symmetric matrix $P$ such that

$$
\left[\begin{array}{cc}
\bar{A}^{\prime} P+P \bar{A} & P \bar{B} \\
\bar{B}^{\prime} P & 0
\end{array}\right]+\left[\begin{array}{c}
\bar{C}^{\prime} \\
\bar{D}^{\prime}
\end{array}\right] W\left[\begin{array}{cc}
\bar{C} & \bar{D}
\end{array}\right]<0
$$


Under the additional assumption that $P>0$, and noting that because $A_{H} \in \mathbb{R}^{n \times n}$, it follows that $P \in \mathbb{R}^{2 n \times 2 n}$ and that $P$ is nonsingular. Hence, partitioning $P$ and $Q:=P^{-1}$ into sub-matrices of dimension $n \times n$

$$
\left[\begin{array}{ll}
Q_{11} & Q_{12} \\
Q_{12}^{\prime} & Q_{22}
\end{array}\right]\left[\begin{array}{ll}
\mathbf{P}_{11} & P_{12} \\
P_{12}^{\prime} & P_{22}
\end{array}\right]=\left[\begin{array}{ll}
I & 0 \\
0 & I
\end{array}\right]
$$

and introducing the matrices

$$
\Pi_{1}:=\left[\begin{array}{ll}
Q_{11} & I \\
Q_{12}^{\prime} & 0
\end{array}\right] \quad \Pi_{2}:=\left[\begin{array}{cc}
I & 0 \\
\mathbf{P}_{11} & P_{12}
\end{array}\right]
$$

it follows that using the congruence transformation $\operatorname{diag}\left(\Pi_{1}, I\right)$ on inequality $(24)$ and carrying out some algebra similar to [14] yield matrix inequality

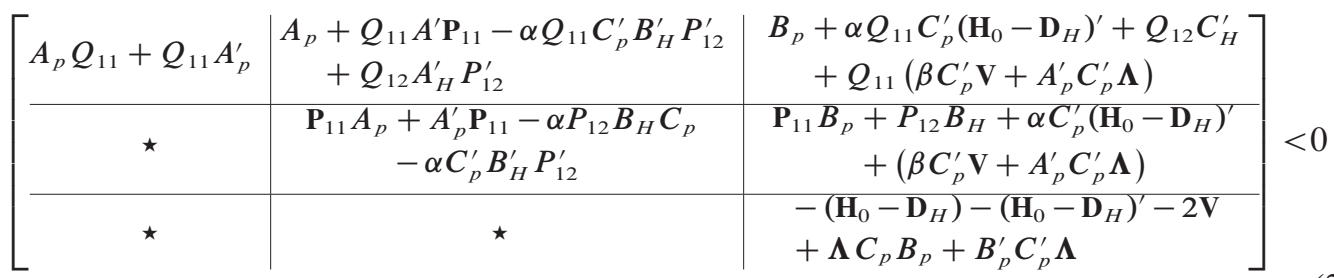

Applying the congruence transformation $\operatorname{diag}\left(Q_{11}^{-1}, I, I\right)=\operatorname{diag}\left(\mathbf{S}_{11}, I, I\right)$ to inequality (27) and defining

$$
\begin{aligned}
& \mathbf{A}_{\mathbf{H}}:=P_{12} A_{H} Q_{12}^{\prime} S_{11} \\
& \mathbf{B}_{\mathbf{H}}:=P_{12} B_{H} \\
& \mathbf{C}_{\mathbf{H}}:=C_{H} Q_{12}^{\prime} S_{11} \\
& \mathbf{D}_{\mathbf{H}}:=D_{H}
\end{aligned}
$$

yield inequality (16).

Part 2: The $\mathcal{L}_{1}$ conditions

The previous part of the proof was similar to that given in $[14,16]$ and was included for completeness. However, in the multivariable case, the multiplier $M(s)$ needs to satisfy the rather more complex ' $\mathcal{L}_{1}$ ' conditions in inequalities (5) and (6). These need more careful consideration than the scalar case presented in [14]. First note that if

$$
\left\|H_{i j}\right\|_{1} \leqslant \xi_{i j}, \quad \xi_{i j}>0 \quad \forall i, j \in\{1, \ldots, m\}
$$

sufficient conditions for inequalities (5) and (6) to hold are given by

$$
\begin{aligned}
& \mathbf{H}_{0, i i} \geqslant \sum_{j=1, j \neq i}^{m}\left|\mathbf{H}_{0, i j}\right|+\sum_{j=1}^{m} \xi_{i j} \forall i \in\{1, \ldots, m\} \\
& \mathbf{H}_{0, i i} \geqslant \sum_{j=1, j \neq i}^{m}\left|\mathbf{H}_{0, j i}\right|+\sum_{j=1}^{m} \xi_{j i} \quad \forall i \in\{1, \ldots, m\}
\end{aligned}
$$

Defining

$$
\gamma_{r, i}:=\sum_{j=1}^{m} \xi_{i j} \geqslant 0
$$




$$
\gamma_{c, i}:=\sum_{j=1}^{m} \xi_{j i} \geqslant 0
$$

inequalities (33) and (34) become

$$
\begin{aligned}
& \mathbf{H}_{0, i i} \geqslant \sum_{j=1, j \neq i}^{m}\left|\mathbf{H}_{0, i j}\right|+\gamma_{r, i} \quad \forall i \in\{1, \ldots, m\} \\
& \mathbf{H}_{0, i i} \geqslant \sum_{j=1, j \neq i}^{m}\left|\mathbf{H}_{0, j i}\right|+\gamma_{c, i} \quad \forall i \in\{1, \ldots, m\}
\end{aligned}
$$

Thus, sufficient conditions for inequalities (5) and (6) to hold are given by inequalities (32), (37) and (38).

Direct application of Lemma 2 implies that inequalities (37) and (38) hold if and only if inequalities (19)-(22) in the Theorem statement hold.

Furthermore, a sufficient condition for inequalities (32) to hold is given by direct application of Lemma 1; that is, inequalities (13) and (14) must hold. Note that there are problematic products of matrix variables $Y_{j}$ and the multiplier state-space matrices $A_{H}$ and $B_{H}$. To remove these products, and again, at the price of some conservatism, let $Y_{j}:=P_{22} \quad \forall j \in\{1, \ldots, m\}$. This then gives the inequalities:

$$
\begin{gathered}
{\left[\begin{array}{cc}
A_{H}^{\prime} P_{22}+P_{22} A_{H}+\lambda_{j} P_{22} & P_{22} B_{H, j} \\
\star & -\mu_{j}
\end{array}\right]<0} \\
{\left[\begin{array}{ccc}
\lambda_{j} P_{22} & 0 & C_{H, i}^{\prime} \\
\star & \left(\xi_{i j}-\mu_{j}\right) & D_{H, i j}^{\prime} \\
\star & \star & \xi_{i j}
\end{array}\right] \geqslant 0}
\end{gathered}
$$

Furthermore, applying the congruence transformation $\operatorname{diag}\left(Q_{12}, I\right)$ to each of the $j$ inequalities (39) gives the $j$ inequalities

$$
\left[\begin{array}{cc}
Q_{12} A_{H}^{\prime} P_{22} Q_{12}^{\prime}+Q_{12} P_{22} A_{H} Q_{12}^{\prime}+\lambda_{j} Q_{12} P_{22} Q_{12}^{\prime} & Q_{12} P_{22} B_{H, j} \\
\star & -\mu_{j}
\end{array}\right]<0
$$

Next, from (25), it follows that $Q_{12} P_{22}=-Q_{11} P_{12}$. Using the inequalities in (41) and noting further from (25) that $Q_{11} P_{12} Q_{12}^{\prime}=Q_{11}\left(I-P_{11} Q_{11}\right)$ the $j$ inequalities are obtained

$$
\left[\begin{array}{cc}
-Q_{12} A_{H}^{\prime} P_{12}^{\prime} Q_{11}-Q_{11} P_{12} A_{H} Q_{12}^{\prime}-\lambda_{j}\left(Q_{11}-Q_{11} \mathbf{P}_{11} Q_{11}\right) & -Q_{11} P_{12} B_{H, j} \\
\star & -\mu_{j}
\end{array}\right]<0
$$

Then, using the congruence transformation $\operatorname{diag}\left(Q_{11}^{-1},-I\right)=\operatorname{diag}\left(S_{11},-I\right)$ yields

$$
\left[\begin{array}{cc}
-\mathbf{S}_{11} Q_{12} A_{H}^{\prime} P_{12}^{\prime}-P_{12} A_{H} Q_{12}^{\prime} \mathbf{S}_{11}-\lambda_{j}\left(\mathbf{S}_{11}-\mathbf{P}_{11}\right) & P_{12} B_{H, j} \\
\star & -\mu_{j}
\end{array}\right]<0
$$

Using (28)-(29) then yields the inequalities (17) in the theorem. In a similar way, applying the congruence transformation diag $\left(Q_{11}^{-1} Q_{12}, I, I\right)$ to each of the inequalities (40) gives after similar working to the above

$$
\left[\begin{array}{ccc}
\lambda_{j}\left(\mathbf{S}_{11}-\mathbf{P}_{11}\right) & 0 & \mathbf{S}_{11} Q_{12} C_{H, i}^{\prime} \\
\star & \xi_{i j}-\mu_{j} & D_{H, i j}^{\prime} \\
\star & \star & \xi_{i j}
\end{array}\right] \geqslant 0
$$

Making these inequalities strict and recalling (28)-(31) then yield inequality (18). 
Part 3: Positive definiteness of $P$

In Part 1 of the proof, it was assumed that $P>0$. Here, we show that satisfaction of the LMIs indeed guarantees that this is indeed the case. Note that $P>0$ is equivalent to $\Pi_{1}^{\prime} P \Pi_{1}>0$, which can be written as

$$
\Pi_{1}^{\prime} P \Pi_{1}=\left[\begin{array}{cc}
Q_{11} & I \\
I & \mathbf{P}_{11}
\end{array}\right]>0
$$

This is equivalent, by the Schur complement, to $\mathbf{P}_{11}-\mathbf{S}_{11}>0$, which is guaranteed by inequality (18).

\section{Remark 6}

- Conservatism: Compared with the SISO case described in [14], there are more potential sources of conservatism in the multivariable results derived here. As with the SISO case, conservatism is introduced by the restriction that $M(s)$ be stable, causal and of order equal to $P(s)$. However, additional conservatism is introduced by bounding each $\left\|H_{i j}\right\|_{1} \leqslant \xi_{i j}$ : only one such bound is introduced in the SISO case. Additionally, to obtain conditions that are convex, it was necessary to stipulate $Y_{j}=P_{22} \forall j$, which introduces yet more conservatism into the results. As with the SISO case, the goal here is to trade conservatism with tractability: again, we note that in its original form (Theorem 1), the search over $M(s)$ is an infinite-dimensional optimisation problem and thus very difficult to solve. Noting the remarks in [26], however, the conservatism in the ' $\mathcal{L}_{1}$ ' conditions can sometimes detrimentally influence conservatism.

- Convexity: As with the SISO case, a remark about convexity is warranted. Note that the inequalities in Theorem 1 are linear for fixed $\lambda_{j}$ and for given sector/slope bounds. Although it was initially thought that $\lambda_{j}$ was not too influential on the results ([14]), recent work has shown that this is not to be the case ([26]) and thus some search over $\lambda_{j}$ appears to be beneficial. When the slope/sector bounds are unknown, it appears reasonable to fix $\beta=\kappa \alpha$, and then Theorem 1 reduces to an LMI plus a bisection over $\alpha$ and a search over $\lambda_{j}$.

\subsection{Related results}

Theorem 2 applies to nonlinearities of the class $\mathcal{N}_{\alpha / \beta}^{R S}$; that is, the sector and slope bounds are not necessarily the same. As observed earlier, in certain nonlinearities of interest, the sector and slope bounds are identical. In this case, it is natural to ponder the existence of improved results. In fact, if $\Phi \in \mathcal{N}_{\alpha / \alpha}^{R S}$ (sector bound given by slope restriction), the Circle IQC used in the derivation of Theorem 2 can be relaxed.

Lemma 3

Let $\Phi(.) \in \mathcal{N}_{\alpha / \alpha}^{R S}$, and let $u=\Phi(y)$; then, the following inequalities hold:

(i)

$$
\Phi(y)^{\prime} V(\alpha y-\Phi(y)) \geqslant 0 \quad \forall y \in \mathbb{R}^{m}
$$

(ii)

$$
\int_{-\infty}^{\infty}\left[\begin{array}{l}
y(j \omega) \\
u(j \omega)
\end{array}\right]^{*}\left[\begin{array}{cc}
0 & \alpha V \\
\alpha V & -2 V
\end{array}\right]\left[\begin{array}{l}
y(j \omega) \\
u(j \omega)
\end{array}\right] \geqslant 0 \quad \forall u \in \mathcal{L}_{2 e}^{m}
$$

where $V=V^{\prime}$ is such that $V_{i i} \geqslant \sum_{j=1, j \neq i}^{m}\left|V_{i j}\right| \quad \forall i$.

Proof

Item (i) has its origins in the work of [21] who stated it for the case where $\mathcal{N}_{1 / \star}^{R S}$. A more general proof is given here. First, let $\Pi(y):=\alpha y-\Phi(y)$, with each element of $\Pi($.$) given by$ $\pi\left(y_{i}\right)=\alpha y_{i}-\phi\left(y_{i}\right)$; then, inequality (i) is equivalent to 


$$
\begin{aligned}
\Phi(y)^{\prime} V \Pi(y) & =\sum_{i=1}^{m} \sum_{j=1}^{m} \phi\left(y_{i}\right) V_{i j} \pi\left(y_{j}\right) \\
& =\sum_{i=1}^{m} \phi_{i} V_{i i} \pi_{i}+\sum_{i=1}^{m} \sum_{j=1, j \neq i}^{m} \phi_{i} V_{i j} \pi_{j} \\
& =\sum_{i=1}^{m}\left(\phi_{i} \pi_{i} V_{i i}+\sum_{j=1, j \neq i}^{m} \phi_{i} V_{i j} \pi_{j}\right) \\
& =\sum_{i=1}^{m}\left(\phi_{i} \pi_{i} V_{i i}+\sum_{j=1, j \neq i}^{m} \phi_{i} \pi_{j}\left|V_{i j}\right| \operatorname{sign}\left(V_{i j}\right)\right)
\end{aligned}
$$

where the shorthand $\phi_{i}:=\phi\left(y_{i}\right)$ and $\pi_{i}:=\pi\left(y_{i}\right)$ has been used. Now, as $\phi_{i} \pi_{i} \geqslant 0$ and as $V_{i i} \geqslant \sum_{j=1, j \neq i}^{m}\left|V_{i j}\right|$, it follows that

$$
\begin{aligned}
\Phi(y)^{\prime} V \Pi(y) & \geqslant \sum_{i=1}^{m}\left(\phi_{i} \pi_{i} \sum_{j=1, j \neq i}^{m}\left|V_{i j}\right|+\sum_{j=1, j \neq i}^{m} \phi_{i} \pi_{j}\left|V_{i j}\right| \operatorname{sign}\left(V_{i j}\right)\right) \\
& =\sum_{i=1}^{m} \sum_{j=1, j \neq i}^{m}\left|V_{i j}\right|\left(\phi_{i} \pi_{i}+\operatorname{sign}\left(V_{i j}\right) \phi_{i} \pi_{j}\right) \\
& =\sum_{i=1}^{m} \sum_{j=1, j \neq i}^{m}\left|V_{i j}\right| \phi_{i}\left(\pi_{i}+\operatorname{sign}\left(V_{i j}\right) \pi_{j}\right)
\end{aligned}
$$

By symmetry of $V$, this becomes

$$
\begin{aligned}
& =\frac{1}{2} \sum_{i=1}^{m} \sum_{j=1, j \neq i}^{m}\left|V_{i j}\right|\left(\phi_{i}\left(\pi_{i}+\operatorname{sign}\left(V_{i j}\right) \pi_{j}\right)+\phi_{j}\left(\pi_{j}+\operatorname{sign}\left(V_{j i}\right) \pi_{i}\right)\right) \\
& =\frac{1}{2} \sum_{i=1}^{m} \sum_{j=1, j \neq i}^{m}\left|V_{i j}\right|\left(\phi_{i}+\operatorname{sign}\left(V_{i j}\right) \phi_{j}\right)\left(\pi_{i}+\operatorname{sign}\left(V_{i j}\right) \pi_{j}\right)
\end{aligned}
$$

This expression will be non-negative if $\phi($.$) and \pi($.$) are monotonically non-decreasing functions.$ Note that as $\phi($.$) is slope restricted in [0, \alpha]$, it is monotonically non-decreasing. Furthermore, because $\pi_{i}=\alpha\left(y_{i}\right)-\phi\left(y_{i}\right)$, monotonicity of $\pi$ holds if the following expression is non-negative for all $y_{1}, y_{2} \in \mathbb{R}$ :

$$
\begin{aligned}
\chi\left(y_{1}, y_{2}\right) & :=\left(\pi\left(y_{1}\right)-\pi\left(y_{2}\right)\right)\left(y_{1}-y_{2}\right) \\
& =\left(\alpha\left(y_{1}-y_{2}\right)-\left(\phi\left(y_{1}\right)-\phi\left(y_{2}\right)\right)\right)\left(y_{1}-y_{2}\right) \\
& =\left(\alpha-\frac{\phi\left(y_{1}\right)-\phi\left(y_{2}\right)}{y_{1}-y_{2}}\right)\left(y_{1}-y_{2}\right)^{2}
\end{aligned}
$$

However, as $\phi \in \partial[0, \alpha]$ by assumption, then $\chi\left(y_{1}, y_{2}\right) \geqslant 0$, and hence $\pi($.$) is monotonically non-$ decreasing. In turn, this implies that $\Phi(y)^{\prime} V \Pi(y)$ is non-negative and hence inequality (i) holds. It is easy to see that inequality (i) implies inequality (ii) and hence the proof is complete.

Therefore if $\Phi(.) \in \mathcal{N}_{\alpha / \alpha}^{R S}$, that is, if the slope restriction and sector bound are identical, $\Phi($. satisfies the IQC (2) defined by $\Pi(j \omega)=\Pi_{\alpha}^{C-D D}(j \omega)$ with

$$
\Pi_{\alpha}^{C-D D}(j \omega)=\left[\begin{array}{cc}
0 & \alpha V \\
\alpha V & -2 V
\end{array}\right]
$$

where $V=V^{\prime}$ is such that $V_{i i} \geqslant \sum_{j=1, j \neq i}^{m}\left|V_{i j}\right| \quad \forall i$. This condition on $V$ is weaker than the requirement for $V$ to be diagonal, and lower conservatism in the results may be expected. 
Thus, in the case that $\Phi(.) \in \mathcal{N}_{\alpha / \alpha}^{R S}$, it satisfies the IQC defined by $\Pi=\tilde{\Pi}_{\alpha / \alpha}^{R S}=\Pi_{\alpha}^{Z F}+\Pi^{P}+$ $\Pi_{\alpha}^{C-D D}$. In a similar way to that described in the previous subsection, the following theorem can then be proved.

\section{Theorem 3}

Assume that $P(s) \in \mathcal{R} \mathcal{H}_{\infty}^{m \times m}$ with $D_{p} \equiv 0$ and that $\Phi(.) \in \mathcal{N}_{\alpha / \alpha}^{\mathrm{RS}}$. Then, the system in Figure 1 is stable if there exist positive definite matrices $\mathbf{S}_{\mathbf{1 1}}, \mathbf{P}_{\mathbf{1 1}}, \mathbf{V}$, a diagonal matrix $\boldsymbol{\Lambda}$, positive scalars $\lambda_{j}, \mu_{j}, \xi_{i j}$, unstructured matrices $\mathbf{A}_{\mathbf{H}}, \mathbf{B}_{\mathbf{H}}, \mathbf{C}_{\mathbf{H}} \mathbf{D}_{\mathbf{H}}, \mathbf{H}_{0}$ and $\mathbf{R}$ such that inequalities (16)-(22) are satisfied, and in addition,

$$
\mathbf{V}_{i i} \geqslant \sum_{j=1, j \neq i}^{m}\left|\mathbf{V}_{i j}\right| \quad \forall i
$$

\section{Remark 7}

Theorems 2 and 3 make use of the Popov multiplier $\Pi^{P}$ in their derivations. From a state-space perspective, this requires the linear subsystem $P(s)$ to be strictly proper (i.e. $D_{p} \equiv 0$ ). If the linear subsystem is not strictly proper, then results based on just the Zames-Falb multiplier may be derived. In this case, the 'passivity' inequality in Proposition 2 of [14] can be combined with inequalities (17)-(22) from Theorem 2 to obtain a direct multivariable extension of [14]. However, it has been noted $([16,17])$ that the inclusion of a Popov term appears to offer some useful numerical flexibility - this will be demonstrated in the next section.

\section{EXAMPLES}

This section compares the results derived here to what appears to be the state-of-the-art in the literature, namely those of [4]. Note that the results of [4] incorporate, as special cases, the Popov criterion and several other stability criteria. It has already been demonstrated that the results of [4] are less conservative than those of Haddad and Kapila [1], Suykens et al. [3] and Chen and Wen [2]. As noted in $[4,14]$, Park's method is also convex, making it relatively easy to compute a solution. In order to apply both the results here and the results of Park, it is necessary to stipulate a relationship between the sector bounds and the slope restrictions. ${ }^{\ddagger}$ For simplicity, we let $\Phi(.) \in \mathcal{N}_{\alpha / \alpha}^{R S}$ and attempt to maximise the value of $\alpha$ for which the relevant criteria guarantee stability. It is important to note that the linear constant gain $\alpha I \in \mathcal{N}_{\alpha / \alpha}^{R S}$, so an upper bound on the maximum achievable $\alpha$ can be determined by simply searching for the maximum $\alpha$ such that the eigenvalues of $A_{p}+\alpha B_{p} C_{p}$ have strictly negative real part. This is important because this gives one an idea of how conservative the results derived here are.

Table I shows a comparison of the maximum sector/slope sizes obtained using the results of Theorem 3, Park's results [4] and the upper bound, for a number of examples. Some of these examples have been taken from the literature, but most have been randomly generated. Rather than giving a long list of state-space matrices in this paper, the reader is directed to [27] for Matlab code for the generation of the examples and results. The values of $\lambda=\lambda_{j} \forall i \in\{1, \ldots, m\}$ are also given: some limited search of $\lambda$ was performed, but these values should not be considered optimal in any way. The results calculated using Theorem 3 all use a proper (rather than strictly proper) ZamesFalb multiplier, which seems to be useful in numerical routines, despite the assertion that it should not [26]. Two cases of Theorem 3 are given: the first without the Circle and Popov terms, that is, $\Pi=\Pi^{Z F}$; and the second with the Circle and Popov terms, that is, $\Pi=\Pi^{Z F}+\Pi^{C-D D}+\Pi^{P}$.

The largest slope/sector sizes for which stability holds for a given example are highlighted in yellow in Table I. The results of [4] give the largest sector sizes for two of the SISO examples used in that paper (examples 1 and 3 in this paper); they also provide the largest sector sizes in the case of examples 28, which is a carefully constructed MIMO version of Example 1. For examples 2, 5,

This is because it is only realistic to maximise either the sector bound or the slope restriction and not both. 
Table I. Maximum slope/sector sizes for stability when $\alpha=\beta$.

\begin{tabular}{|c|c|c|c|c|c|}
\hline \multirow[b]{2}{*}{ Example } & \multirow[b]{2}{*}{ Notes } & \multicolumn{4}{|c|}{ Maximum $\alpha$} \\
\hline & & $\Pi^{Z F}$ & $\begin{array}{c}\text { Theorem } 3 \\
\Pi^{Z F}+\Pi^{P}+\Pi^{C-D D}\end{array}$ & Park [4] & $\begin{array}{l}\text { Linear } \\
\text { upper bound }\end{array}$ \\
\hline 1 & [4] $n=3, m=1 ; \lambda=0.32 / 831.2$ & 2.4283 & 3.5071 & 4.5894 & 4.5894 \\
\hline 2 & [4] $n=3, m=1 ; \lambda=3.6$ & 1.0894 & 1.0894 & 1.0894 & 1.0894 \\
\hline 3 & [4] $n=4, m=1 ; \lambda=0.09 / 482.7$ & 0.7063 & 0.77903 & 0.78826 & $\infty$ \\
\hline 4 & {$[14] n=4, m=1 ; \lambda=0.54 / 1.553$} & 0.85328 & 1.0864 & 0.70832 & 3.5 \\
\hline 5 & [4] $n=3, m=2 ; \lambda=0.14$ & 0.23235 & 0.23255 & 0.23255 & 0.23255 \\
\hline 6 & {$[27] n=3, m=2 ; \lambda=1.2$} & 5.708 & 5.9483 & 5.9483 & 5.9483 \\
\hline 7 & {$[13] n=8, n=2 ; \lambda=10^{-5}$} & 1.1248 & 1.1257 & 1.1257 & 1.1257 \\
\hline 8 & [4] $n=9, m=3 ; \lambda=10^{-5}$ & 0.92371 & 0.92371 & 0.92371 & 0.92371 \\
\hline 9 & {$[27] n=9, m=3 ; \lambda=10^{-8}$} & 0.76442 & 0.76442 & 0.76442 & 0.76442 \\
\hline 10 & {$[27] n=4, m=3 ; \lambda=10^{-5}$} & 1.1017 & 3.3299 & 2.6361 & 4.158 \\
\hline 11 & {$[27] n=4, m=3 ; \lambda=0.09$} & 0.0636 & 0.09075 & 0.09075 & 0.09075 \\
\hline 12 & [27] $n=4, m=3 ; \lambda=0.5$ & 3.1965 & 7.7274 & 6.5424 & 8.3093 \\
\hline 13 & {$[27] n=4, m=3 ; \lambda=10^{-5}$} & 0.1943 & 0.21081 & 0.19612 & 0.21081 \\
\hline 14 & {$[27] n=4, m=3 ; \lambda=10^{-5}$} & 0.67767 & 0.77276 & 0.54276 & 0.93424 \\
\hline 15 & {$[27] n=6, m=3 ; \lambda=0.05$} & 0.0392 & 0.04029 & 0.03835 & 0.04029 \\
\hline 16 & {$[27] n=6, m=3 ; \lambda=10^{-5}$} & 0.07674 & 0.08273 & 0.06204 & 0.08273 \\
\hline 17 & {$[27] n=6, m=4 ; \lambda=10^{-5}$} & 0.08692 & 0.08692 & 0.08447 & 0.08692 \\
\hline 18 & {$[27] n=6, m=4 ; \lambda=0.5$} & 0.22309 & 0.33081 & 0.18121 & 0.91517 \\
\hline 19 & {$[27] n=6, m=4 ; \lambda=0.15$} & 0.43229 & 0.48048 & 0.22658 & 0.82015 \\
\hline 20 & {$[27] n=6, m=4 ; \lambda=0.5$} & 0.25462 & 0.31783 & 0.23540 & 0.35339 \\
\hline 21 & [27] $n=6, m=4 ; \lambda=1$ & 0.68574 & 0.80657 & 0.65797 & 0.83174 \\
\hline 22 & {$[27] n=8, m=4 ; \lambda=0.1$} & 0.00169 & 0.00191 & 0.00103 & 0.002 \\
\hline 23 & {$[27] n=8, m=2 ; \lambda=10$} & 0.02497 & 0.02497 & 0.02322 & 0.02497 \\
\hline 24 & {$[27] n=8, m=2 ; \lambda=0.4$} & 0.12092 & 0.12411 & 0.11855 & 0.12531 \\
\hline 25 & {$[27] n=8, m=2 ; \lambda=10^{-3}$} & 0.00427 & 0.00428 & 0.00428 & 0.00428 \\
\hline 26 & {$[27] n=8, m=2 ; \lambda=0.1$} & 0.02709 & 0.02709 & 0.02668 & 0.02709 \\
\hline 27 & {$[27] n=8, m=2 ; \lambda=0.05$} & 0.00407 & 0.00415 & 0.00364 & 0.00496 \\
\hline 28 & [27]: MIMO Ex $1 ; \lambda=0.32 / 831.2$ & 1.7035 & 1.9757 & 3.7508 & 3.7508 \\
\hline
\end{tabular}

Numerical values accurate to five significant figures.

7, 8 and 9, both the method of [4] and Theorem 3 (with and without the Popov multiplier) are able to guarantee stability for the slope/sector size predicted by linear analysis. For examples 6, 11 and 25, the method of [4] and Theorem 3 are also able to guarantee stability for the same slope/sector size predicted by linear analysis, but now the Popov multiplier must be included in Theorem 3 in order to obtain these slope/sector sizes. For the remainder of the examples, it appears that the largest sector/slope sizes for which stability holds are guaranteed by Theorem 3 (sometimes with and without the Popov multiplier). These results clearly show that, in many examples, application of Theorem 3 leads to a less conservative estimate of the sector/slope size the system is able to tolerate before instability results.

Note that, apart from example 28, it appears that the results given here are typically equal or superior to [4] in the MIMO cases. It is interesting to understand why this might be the case, and it is probably because, in the SISO case, it is the conservatism of the $\mathcal{L}_{1}$ inequalities that 'limits' the effectiveness of Theorem 2, whereas in the MIMO case, the 'passivity' condition (inequality (27)) can often be difficult to satisfy: With the results of Theorem 2, there is more freedom in satisfying this condition than that in the equivalent condition in [4].

\section{CONCLUSION}

This paper has proposed a new, reasonably tractable approach for assessing the stability of multivariable feedback interconnections consisting of a linear part and a nonlinearity in which all elements have identical sector bounded, slope-restricted characteristics. Various examples, both 
from the literature and randomly generated, have shown the effectiveness of the results. The SISO versions of these results ([14]) found application in anti-windup compensator design [28]; it is envisaged that the results here will be useful in this and other applications.

\section{APPENDIX A: PROOF OF LEMMA 1}

The proof follows in a very similar way to [24]; the difference is that here the desire is to ensure bounds on the component-wise $\mathcal{L}_{1}$ gains. First of all, note that a state-space realisation for each $H_{i j}(s)$ is given by

$$
H_{i j}(s) \sim\left[\begin{array}{c|c}
A_{H} & B_{H, j} \\
\hline C_{H, i} & D_{H, i j}
\end{array}\right]
$$

Define $z_{i}:=H_{i j}(s) w_{j}$, and note that if $\left\|z_{i}\right\| \leqslant \xi_{i j}$ for all $\left\|w_{j}\right\|=1$, then $\left\|H_{i j}\right\|_{1} \leqslant \xi_{i j}$.

Next, define the Lyapunov function candidate $V_{j}(x):=x^{\prime} Y_{j} x$. Then, pre-multiplying and post-multiplying inequality (13) in Lemma 1 by $\left[\begin{array}{ll}x^{\prime} & w_{j}\end{array}\right]^{\prime}$ and its transpose, respectively, imply that

$$
\frac{d}{d t}\left(V_{j}(x)\right)+\lambda_{j} V_{j}(x)-\mu_{j} w_{j}^{\prime} w_{j}<0
$$

Noting that $\left\|w_{j}\right\|=1$, this implies that $\dot{V}_{j}(x)<0$ when $V_{j}(x) \geqslant \mu_{j} / \lambda_{j}$. This means that with $V_{j}(x(0))=0$, an upper bound on $V_{j}(x)$ is given by

$$
V_{j}(x) \leqslant \frac{\mu_{j}}{\lambda_{j}} \Leftrightarrow \lambda_{j} V_{j}(x)-\mu_{j} \leqslant 0
$$

Next, note that inequality (14) implies, via the Schur complement, that

$$
\left[\begin{array}{cc}
\lambda_{j} Y_{j} & 0 \\
0 & \xi_{i j}-\mu_{j}
\end{array}\right] \geqslant \frac{1}{\xi_{i j}}\left[\begin{array}{c}
C_{H, i}^{\prime} \\
D_{H, i}^{\prime}
\end{array}\right]\left[\begin{array}{ll}
C_{H, i}^{\prime} & D_{H, i}^{\prime}
\end{array}\right]
$$

Pre-multiplying and post-multiplying by $\left[\begin{array}{ll}x & w_{j}\end{array}\right]^{\prime}$ and its transpose then imply that

$$
\lambda_{j} V_{j}(x)+w_{j}\left(\xi_{i j}-\mu_{j}\right) w_{j} \geqslant \frac{1}{\xi_{i j}} z_{i}^{2}
$$

Noting $\lambda_{j} V_{j}(x)-\mu_{j} \leqslant 0$ then implies that $z_{i}^{2} \leqslant \xi_{i j}^{2}$, which proves the result.

\section{REFERENCES}

1. Haddad WM, Kapila V. Absolute stability criteria for multiple slope-restricted nonlinearities. IEEE Transactions on Automatic Control 1995; 40(2):361-365.

2. Chen. X, Wen T. Robustness analysis of LTI systems with structured incrementally sector bounded nonlinearities. Proc. of the American Control Conference, Seattle, USA, 1995; 3883-3887.

3. Suykens JAK, Vandewalle J, De Moor B. An absolute stability criterion for the Lur'e problem with sector and slope restricted nonlinearities. IEEE Transactions on Circuits and Systems 1998; 45(9):1007-1009.

4. Park PG. Stability criteria of sector and slope restricted Lur'e systems. IEEE Transactions on Automatic Control 2002; 47(2):308-313.

5. Megretski AV, Ranzter A. System analysis via integral quadratic constraints. IEEE Transactions on Automatic Control 1997; 42(6):819-830.

6. Desoer CA, Vidyasagar M. Feedback Systems: Input-Output Properties. SIAM Classics in Applied Mathematics: Philadelphia, 1975.

7. Zames G, Falb PL. Stability conditions for systems with monotone and slope restricted nonlinearities. SIAM Journal of Control 1968; 6(1):89-108.

8. Safonov MG, Kulkarni VV. Zames-Falb multipliers for MIMO nonlinearities. International Journal of Robust and Nonlinear Control 2000; 10(10):1025-1038.

9. Kulkarni VV, Safonov MG. All multipliers for repeated monotone nonlinearities. IEEE Transactions on Automatic Control 2002; 47(7):1209-1212.

10. Mancera R, Safonov MG. All stability multipliers for repeated MIMO nonlinearities. Systems and Control Letters 2005; 54:389-397. 
11. Altshuller DA. Delay-integral-quadratic constraints and stability multipliers for systems with MIMO nonlinearities. IEEE Transactions on Automatic Control 2011; 56(4):738-747.

12. Megretski AV, Kao CY, Jonsson UT, Rantzer A. A guide to IQC $\beta$ : a Matlab toolbox for robust stability and performance analysis. Technical Report, MIT, 2004.

13. D'Amato FJ, Rotea MA, Megretski AV, Jonsson UT. New results for analysis of systems with repeated nonlinearities. Automatica 2001; 37(6):739-747.

14. Turner MC, Kerr ML, Postlethwaite I. On the existence of stable, causal multipliers for systems with slope-restricted nonlinearities. IEEE Transactions on Automatic Control 2009; 54(11):2697-2702.

15. Turner MC, Kerr ML, Postlethwaite I, Sofrony J. $\mathcal{L}_{2}$ gain bounds for systems with slope restricted nonlinearities. Proc. of the American Control Conference, Baltimore, 2010; 2671-2676.

16. Turner MC, Kerr ML. $\mathcal{L}_{2}$ gain bounds for systems with slope-restricted and sector-bounded nonlinearities. International Journal of Robust and Nonlinear Control 2012; 22(13):1505-1521.

17. Carrasco J, Maya-Gonzalez, Lanzon A, Heath WP. LMI search for rational anticausal Zames-Falb multipliers. Proc. IEEE Conf. Decision and Control, Hawaii, 2012; 7770-7775.

18. Chang M, Mancera R, Safonov MG. Computation of Zames-Falb multipliers revisited. IEEE Transactions on Automatic Control 2012; 57(4):1024-1029.

19. Khalil HK. Nonlinear Systems. Prentice Hall: New Jersey, 1996.

20. Turner MC, Kerr ML, Sofrony J. On stability analysis for systems with repeated slope-restricted nonlinearities. Proc. American Control Conference, Montreal, 2012; 2589-2594.

21. Chu YC, Glover K. Bounds of the induced norm and model reduction errors for systems with repeated scalar nonlinearities. IEEE Transactions on Automatic Control 1999; 44(3):471-483.

22. Jonsson U. Stability analysis with Popov multipliers and integral quadratic constraints. Systems and Control Letters 1997; 31:85-92.

23. Mancera R, Safonov MG. An algorithm to compute multipliers for repeated monotone nonlinearities. Proc. IEEE Conference on Decision and Control, Las Vegas, USA, 2002; 4162-4166.

24. Scherer C, Gahinet P, Chilali M. Multiobjective output feedback control via LMI optimisation. IEEE Transactions on Automatic Control 1997; 42(7):896-911.

25. Rantzer A. On the Kalman-Yakubovich-Popov lemma. Systems and Control Letters 1996; 28(1):7-10.

26. Carrasco J, Heath WP, Li G, Lanzon A. Comments on 'On the existence of stable, causal multipliers for systems with slope-restricted nonlinearities'. IEEE Transactions on Automatic Control 2012; 57(9):2422-2428.

27. Matlab software for repeated scalar nonlinearity analysis, 2013. http://www.le.ac.uk/eg/mct6 [Accessed on 25th November 2013].

28. Kerr M, Turner MC, Villota E, Jayasuriya S, Postlethwaite I. A robust anti-windup design procedure for SISO systems. International Journal of Control 2011; 84(2):351-369. 